\title{
Nucleolin: a prognostic marker in cancer?
}

\author{
Philippe Bouvet ${ }^{1,2 *}$
}

${ }^{1}$ Cancer Cell Plasticity Department, Centre de Recherche en Cancérologie de Lyon, Université de Lyon, UMR INSERM 1052 CNRS 5286, Centre Léon Bérard, Lyon 69008, France

2Ecole Normale Supérieure de Lyon, Université de Lyon, Lyon 69342, France

*Correspondence to: Philippe Bouvet, Cancer Cell Plasticity Department, Cancer Research Center of Lyon, University of Lyon, UMR INSERM 1052 CNRS 5286 , Léon Bérard Center, Lyon 69008, France; Tel: +33627446212; E-mail: pbouvet@ens-lyon.fr

Received: July 08, 2019; Accepted: July 16, 2019; Published: August 11, 2019;

\begin{abstract}
Nucleolin, a major protein of the nucleolus which is required for cell proliferation is the subject of numerous studies in the field of oncology. Many studies suggest that the level of expression of nucleolin and its cellular localization could be an indication of the clinical outcome in several cancers. Quite logically for a protein required for the cancer cells to increased protein synthesis, a correlation between the high level of nucleolin expression and the poor prognosis of the patients is often reported. Unexpectedly, in several studies it was also found that a low nucleolin expression could be also associated with an unfavorable prognosis. In these commentary I discuss these studies and the possible implications for using nucleolin as a prognostic marker in oncology.
\end{abstract}

Keywords: Nucleolin, Cancer, Prognostic Marker

\section{Nucleolin, an abundant multifunctional protein with multiple cellular localization}

Nucleolin was first described as an abundant phopho-nucleolar protein [1]. The protein is heavily modified post-translationally [2] and can interact with numerous proteins $[3]$ and nucleic acids $[4,5]$. The nucleolar localization of nucleolin suggested that it could be involved in some aspects of ribosome biogenesis as the nucleolar structures are the sites of transcription of the pre-ribosomal RNA and of its assembly with ribosomal proteins to form the pre-ribosomes that are then exported in the cell cytoplasm for the mRNA translation. Indeed, it was shown that nucleolin expression was required for the formation of functional nucleolar structure $[6,7]$. Nucleolin is required for RNA polymerase I transcription $[8,9]$ probably through its chaperone activity that facilitates transcription of chromatin [10-12]. It has been suggested that the transient interaction of nucleolin with the prerRNA during transcription might be involved in the correct folding of the long pre-RNA which is required for the processing in mature ribosomal RNA [13-17] but it remains to be demonstrated if the RNA binding specificity of nucleolin towards pre-rRNA is important as deletion of the RNA binding domains responsible for this specificity do not seems to be crucial for the vital function of nucleolin [7]. By analogy to this co-transcriptional role of nucleolin in pre-rRNA maturation and assembly with proteins, it has been recently suggested that acetylated nucleolin could also be involved in the assembly of pre-catalytic spliceosomes and in splicing reaction [18, 19]. Several reports suggest also that nucleolin could be involved in the regulation of transcription of some ANP polymerase II genes [20-24]. Whether there is a functional link between this RNAPII regulation and the implication in the co-transcriptional splicing of some pre-mRNA remains to be determined. In addition to this fundamental role in the regulation of gene expression, there has been a renewed interest in the study of this protein because it has been discovered that nucleolin is also present on the cell surface. Initially described as a HIV gp120 V3 loop binding protein which could be involved in the binding of HIV particles to CD4(+) cells $[25,26]$, it was then suggested that cell surface nucleolin could be a receptor for diverse ligands [27-29].

Cell surface nucleolin was then described as a marker of endothelial cells in angiogenic blood [30] and this specific localization was associated with cancer cells [31-36]. Whether the localization of nucleolin on the cell surface is specific to the cancerous nature of the cell or is the result of a high proliferation rate of these cells remains to be determined. One major difficulty to study the function of cell surface nucleolin and its link with cancer cells is that the detection of cell surface nucleolin is difficult. Initial studies were performed by incubating cells with a monoclonal antibody ( $\mathrm{mAb} \mathrm{D} 3$ ) at room temperature before paraformaldehyde (PFA) fixation [37]. The beautiful images showing a regular localization of nucleolin on the surface of HeLa cells are very difficult to reproduce with other antinucleolin antibodies. Nevertheless, in other studies, immunoelectron microscopy [38] or cell fractionation [37, 39] confirmed the presence of nucleolin on the cell surface.

\section{Is Nucleolin expression a marker for cancer cells?}

Since several decades, it is known that cells that have a high proliferation rate express more nucleolar proteins (including nucleolin) as reflected by the silver staining method (AgNOR) [40]. It is therefore not surprising that, in general, cancer cells are also characterized by a higher level of detection of AgNOR proteins [41, 
42]. This can be easily explained by the need of the cancer cells to produce more proteins to support the higher proliferation of these cells, and therefore by the need to make more ribosomes to produce these proteins.

Indeed, in many studies, it is described that nucleolin is overexpressed in tumors and cancer cells as detected by RT-QPCR, western blot or immunocytochemistry. For instance, an overexpression of nucleolin was detected in glioblastoma cells [43], hepatocellular carcinoma [44], pancreatic cancer [45], non-small cell lung cancer [46], acute myeloid leukemia [47], breast cancer [48]. However, it is very rare if in these studies the different pools of nucleolin (nucleoplasmic, nucleolar, cytoplasmic and at the cell surface) were discriminated. As the nucleolar pool of nucleolin represents the vast majority of total nucleolin protein, it is very likely that the increased expression of nucleolin results in an increased of this nucleolar pool (which participates to the increased in RNA polymerase I transcription of pre-ribosomal RNA) and also to the other cellular pools. Stating that nucleolin is overexpressed in cancer cells is however an unfortunate simplification found in many research papers and reviews. The real situation is indeed more complicated and finer studies on the level of expression of nucleolin should be done especially using large series of biological samples.

In many studies, the level of expression of nucleolin at the mRNA or protein level is examined in a limited number of biological samples or could even limited to established cell lines representing models for a specific cancer. In these cases, the results should be taken with care, as the expression of nucleolin could greatly fluctuate and be very sensitive to the growth conditions of culture cells.

When large number of samples are used, a wide range of nucleolin fold change is usually observed. Then, it can become a challenge to determine which expression level represent a "low" versus a "high" expression. The discrimination between "low" and "high" can be done only if enough samples are analyzed and if a good control group is also examined at the same time. Then a "low" nucleolin expression may represent the normal expression found in control samples, or an expression that is lower than in the control group.

In human non-small cell lung cancer (NSCLC), immunohistochemistry was used to measure nucleolin expression in tissues from 225 NSCLC patients [46]. Higher expression of nucleolin was observed in $62,8 \%$ of the patients and the authors tried to discriminate the level of nuclear versus cytoplasmic nucleolin expression. If about equal number out of the 225 samples show either a low or high expression of cytoplasmic nucleolin, about $68 \%$ of the tumors had a low expression of nuclear nucleolin (and therefore $32 \%$ had a high expression of the nuclear pool of nucleolin). In that example, higher nucleolin nuclear level was associated with better survival while high level of cytoplasmic nucleolin was associated with poorer prognosis [46]. The same conclusions was drawn in a study on gastric cancer [49]. Among 124 gastrectomy samples, 68,5 \% showed a high nucleolin expression. It was found that a high nucleolar nucleolin expression was associated with a better prognosis while tumors from patients showing a high cytoplasmic detection had the worse prognosis. In most of these studies the elevated total nucleolin expression is associated with a poor prognosis of patients like in gastric cancer, hepatocellular carcinoma, acute myeloid leukemia, non-small cell lung cancer, pancreatic ductal adenocarcinoma [44, 47, 49-51]. Interestingly, in AML, the higher nucleolin mRNA expression level, compared to normal blasts, was associated with a poor survival only in elderly patients, and was found to be an independent marker [47].

The prognostic value of nucleolin expression was analyzed in acute myeloid leukemia (AML). In this study, the $75^{\text {th }}$ percentile was used to form the two group of low and high expression [47] using different series of biological samples or TGCA data sets representing 270 samples in total. In this type of cancer, the nucleolin expression was found to cover a very large dispersion. Using this patient stratification, it was found that high nucleolin expression was associated with a poor survival in elderly patients. In a study on the correlation between nucleolin expression and patient outcome with hepatocellular carcinoma, it was found in a series of 130 patients that about $60 \%$ of tumors expressed "high" nucleolin expression and $40 \%$ a "low" expression [44] with very little explanation on how the cut off was done. The "high" nucleolin expression was then associated with poor prognosis of the patient.

However, it seems that there is not always a strict correlation between the level of nucleolin expression and the aggressiveness of the tumor and the outcome of the prognosis.

In another study in breast cancer, a quartile distribution analysis was first performed and highlighted that two quartiles can be grouped. Therefore, the expression levels could be stratified in three group representing a "low", "medium" and "high" nucleolin expression [48]. Unexpectedly, using this stratification it was found that "low" and "High" expression levels of nucleolin were both markers of poor survival in triple negative breast cancer (TNBC). Interestingly, transcriptomic analysis of these two groups of samples in the TCGA data base revealed different gene expression profiles suggesting that the tumors in these two groups are different allowing a better stratification of TNBC patients. In agreement with these data, in another independent study, it was found by immunohistochemistry on a panel of $70 \mathrm{TNBC}$ that nucleolin was undetectable in about $20 \%$ of these tumor which could correspond to the "low" expression while the expression was "high" in $30 \%$ of the tumors [52]. In this study, the authors did not determine if there was a correlation between this patient stratification based on nucleolin expression and the prognostic outcome.

In a study using 69 biological samples from patients with stage II pancreatic ductal adenocarcinoma (PDAC), the authors determined nucleolin expression using immunohistochemistry and scored the nucleolin expression levels using the median nucleolin labeling index as cut off [50]. They found that low level of nucleolin expression was a marker of poor prognosis $(19.5 \pm 3.3$ months versus $65.2 \pm 16.3$ months for patients having tumor with a higher nucleolin expression).

\section{Conclusion}

In most of the studies, the expression of nucleolin is not homogeneous in the different tumors samples. If overall, the expression of nucleolin seems higher in the majority of the tumors it remains that 
a substantial number of tumors show a low or intermediate expression of nucleolin. In several cases, the tumors showing a low expression have been also associated with a poor prognosis. In addition, the localization of nucleolin expression within the cells (nuclear versus cytoplasmic) seems to be also important to predict the outcome of the patient. It is therefore important to be able to discriminate the level of nucleolin expression compared to normal tissue (which cutt off is used to make the distinction between high and low expression) and to have enough biological samples to be able to draw any conclusion on the prognostic value of nucleolin expression. As recently shown with breast TNBC series, the stratification of patients according to nucleolin expression level could be an additional criteria to choose a therapeutic strategy and to develop clinical trials with available molecules that target nucleolin.

\section{Competing interests}

The authors declare that he has no competing interests.

\section{Funding information}

The research in author team is funded by the Ligue contre le Cancer (Allier and Saone et Loire, France), the Foundation pour la recherche sur le Cancer (ARC) the CNRS, the Ecole Normale Supérieure de Lyon, the Agence Nationale de la Recherche, project ANR Theranuc, ANR-16-CE17-0023. These funding agencies have no roles in the design of the studies, analysis and interpretation of the data, in the writing of the report; and in the decision to submit the paper for publication.

\section{References}

1. Orrick L.R, Olson M.O, Busch H. (1973) Comparison of nucleolar proteins of normal rat liver and Novikoff hepatoma ascites cells by two-dimensional polyacrylamide gel electrophoresis. Proc Natl Acad Sci 1316-1320.

2. Ugrinova I, Petrova M, Chalabi-Dchar M, Bouvet P. (2018) Multifaceted Nucleolin Protein and Its Molecular Partners in Oncogenesis, Adv Protein Chem Struct Biol 133-164.

3. Salvetti A., Coute Y., Epstein A., Arata L., Kraut A. (2016) Nuclear functions of nucleolin through global proteomics and interactomic approaches, J Proteome Res.

4. Abdelmohsen K., Tominaga K., Lee E.K., Srikantan S., Kang M.J. (2011) Enhanced translation by Nucleolin via G-rich elements in coding and non-coding regions of target mRNAs, Nucleic Acids Res 8513-8530.

5. Ginisty H., Sicard H., Roger B., Bouvet P. (1999) Structure and functions of nucleolin, J Cell Sci 761-772.

6. Ugrinova I., Monier K., Ivaldi C., Thiry M., Storck S. (2007) Inactivation of nucleolin leads to nucleolar disruption, cell cycle arrest and defects in centrosome duplication, BMC Mol Biol 66.

7. Storck S., Thiry M., Bouvet P. (2009) Conditional knockout of nucleolin in DT40 cells reveals the functional redundancy of its RNA-binding domains, Biol Cell 153-167.

8. Cong R., Das S., Ugrinova I., Kumar S., Mongelard F. (2012) Interaction of nucleolin with ribosomal RNA genes and its role in RNA polymerase I transcription, Nucleic Acids Res 9441-9454.

9. Durut N., Saez-Vasquez J., Nucleolin: dual roles in rDNA chromatin transcription, Gene 556 (2014) 7-12.

10. Angelov D., Bondarenko V.A., Almagro S., Menoni H., Mongelard F. (2006) Nucleolin is a histone chaperone with FACT-like activity and assists remodeling of nucleosomes, EMBO J 25 1669-1679.

11. Pontvianne F., Abou-Ellail M., Douet J., Comella P., Matia I. (2010) Nucleolin is required for DNA methylation state and the expression of rRNA gene variants in Arabidopsis thaliana, PLoS Genet 6 e1001225.

12. Rickards B., Flint S.J., Cole M.D., LeRoy G. (2007) Nucleolin is required for RNA polymerase I transcription in vivo, Mol Cell Biol 937-948.

13. Allain F.H., Bouvet P., Dieckmann T., Feigon J. (2000) Molecular basis of sequencespecific recognition of pre-ribosomal RNA by nucleolin, EMBO 6870-6881.
14. Bouvet P., Allain F.H., Finger L.D., Dieckmann T., Feigon J. (2001) Recognition of pre-formed and flexible elements of an RNA stem-loop by nucleolin, $J$ Mol Biol 763-775.

15. Bouvet P., Jain C., Belasco J.G., Amalric F., Erard M. (1997) RNA recognition by the joint action of two nucleolin RNA-binding domains: genetic analysis and structural modeling, EMBO J 16 5235-5246.

16. Ginisty H., Amalric F., Bouvet P. (1998) Nucleolin functions in the first step of ribosomal RNA processing, EMBO J 17 1476-1486.

17. Serin G., Joseph G., Faucher C., Ghisolfi L., Bouche G. et al. (1996) Localization of nucleolin binding sites on human and mouse pre-ribosomal RNA, Biochimie 530-538.

18. Das S., Cong R., Shandilya J., Senapati P., Moindrot B. (2013) Characterization of nucleolin K88 acetylation defines a new pool of nucleolin colocalizing with premRNA splicing factors, FEBS Lett 417-424.

19. Ugrinova I., Chalabi-Dchar M., Monier K., Bouvet P. (2019) Nucleolin Interacts and Co-Localizes with Components of Pre-Catalytic Spliceosome Complexes, Sci 133 .

20. Allinne J., Pichugin A., Iarovaia O., Klibi M., Barat A. et al. (2014) Perinucleolar relocalization and nucleolin as crucial events in the transcriptional activation of key genes in mantle cell lymphoma, Blood 123 2044-2053.

21. Cong C., Das S., Bouvet P. (2011) The Multiple Properties and Functions of Nucleolin, Protein Reviews series, Nucleolus, Springer Editor-in-Chief is M. Zouhair Atassi 185.

22. Grinstein E., Du Y., Santourlidis S., Christ J., Uhrberg M. (2007) Nucleolin regulates gene expression in CD34-positive hematopoietic cells, J Biol Chem 12439-12449.

23. Hanakahi L.A., Dempsey L.A., Li M.J., Maizels N. (1997) Nucleolin is one component of the B cell-specific transcription factor and switch region binding protein, LR1, Proc Natl Acad Sci 94 (1997) 3605-3610.

24. Kumar S., Gomez E.C., Chalabi-Dchar M., Rong C., Das S. (2017) Integrated analysis of mRNA and miRNA expression in HeLa cells expressing low levels of Nucleolin, Sci Rep 79017.

25. Callebaut C., Blanco J., Benkirane N., Krust B., Jacotot E. (1998) Identification of V3 loop-binding proteins as potential receptors implicated in the binding of HIV particles to CD4(+) cells, J Biol Chem 21988-21997.

26. Nisole S., Krust B., Callebaut C., Guichard G., Muller S. et al. (1999) Hovanessian A.G., The anti-HIV pseudopeptide HB-19 forms a complex with the cell-surfaceexpressed nucleolin independent of heparan sulfate proteoglycans, $J$ Biol Chem 27875-27884.

27. Harms G., Kraft R., Grelle G., Volz B., Dernedde J. (2001) Identification of nucleolin as a new L-selectin ligand, Biochem J 360 531-538.

28. Said E.A., Krust B., Nisole S., Svab J., Briand J.P. (2002) The anti-HIV cytokine midkine binds the cell surface-expressed nucleolin as a low affinity receptor, $J$ Biol Chem 277 37492-37502.

29. Sinclair J.F., O'Brien A.D., Cell surface-localized nucleolin is a eukaryotic receptor for the adhesin intimin-gamma of enterohemorrhagic Escherichia coli O157:H7, J Biol Chem 277 (2002) 2876-2885.

30. Christian S., Pilch J., Akerman M.E., Porkka K., Laakkonen P. (2003) Nucleolin expressed at the cell surface is a marker of endothelial cells in angiogenic blood vessels, J Cell Biol 163 871-878.

31. Fujiki H., Watanabe T., Suganuma M. (2014) Cell-surface nucleolin acts as a central mediator for carcinogenic, anti-carcinogenic, and disease-related ligands, $J$ Cancer Res Clin Oncol 689-699.

32. Galzio R., Rosati F., Benedetti E., Cristiano L., Aldi S. et al. (2012) Glycosilated nucleolin as marker for human gliomas, J Cell Biochem 113 571-579.

33. Joo E.J., ten Dam G.B., van Kuppevelt T.H., Toida T., Linhardt R.J. et al. (2005) Nucleolin: acharan sulfate-binding protein on the surface of cancer cells, Glycobiology 1-9.

34. Reyes-Reyes E.M., Akiyama S.K. (2008) Cell-surface nucleolin is a signal transducing P-selectin binding protein for human colon carcinoma cells, Exp Cell Res 314 2212-2223.

35. Watanabe T., Hirano K., Takahashi A., Yamaguchi K., Beppu M. et al. (2010) Nucleolin on the cell surface as a new molecular target for gastric cancer treatment, Biol Pharm Bull 796-803.

36. Watanabe T., Tsuge H., Imagawa T., Kise D., Hirano K. (2010) Nucleolin as cell surface receptor for tumor necrosis factor-alpha inducing protein: a carcinogenic factor of Helicobacter pylori, J Cancer Res Clin Oncol 136 911-921.

37. Hovanessian A.G., Puvion-Dutilleul F., Nisole S., Svab J., Perret E. et al. (2000) The cell-surface-expressed nucleolin is associated with the actin cytoskeleton, Exp Cell Res 261 312-328.

38. Dumler I., Stepanova V., Jerke U., Mayboroda O.A., Vogel F. et al. (1999) Urokinase-induced mitogenesis is mediated by casein kinase 2 and nucleolin, Curr Biol 9 1468-1476.

39. Losfeld M.E., Khoury D.E., Mariot P., Carpentier M., Krust B. et al. (2009) The cell surface expressed nucleolin is a glycoprotein that triggers calcium entry into mammalian cells, Exp Cell Res 315 357-369. 
40. Derenzini M., Sirri V., Pession A., Trere D., Roussel P. et al. (1995) HernandezVerdun D., Quantitative changes of the two major AgNOR proteins, nucleolin and protein B23, related to stimulation of rDNA transcription, Exp Cell Res 219 276-282.

41. King J., Thatcher N., Pickering C., Hasleton P. (2006) Sensitivity and specificity of immunohistochemical antibodies used to distinguish between benign and malignant pleural disease: a systematic review of published reports, Histopathology 49 561568 .

42. Pich A., Chiusa L., Margaria E. (2000) Prognostic relevance of AgNORs in tumor pathology, Micron 31 133-141.

43. Balca-Silva J., do Carmo A., Tao H., Rebelo O., Barbosa M. (2018) Nucleolin is expressed in patient-derived samples and glioblastoma cells, enabling improved intracellular drug delivery and cytotoxicity, Exp Cell Res 370 68-77.

44. Guo X., Xiong L., Yu L., Li R., Wang Z. (2014) Increased level of nucleolin confers to aggressive tumor progression and poor prognosis in patients with hepatocellular carcinoma after hepatectomy, Diagn Pathol 175.

45. Gilles M.E., Maione F., Cossutta M., Carpentier G., Caruana L., et al. (2016) Nucleolin Targeting Impairs the Progression of Pancreatic Cancer and Promotes the Normalization of Tumor Vasculature, Cancer Res 76 7181-7193.

46. Xu J.Y., Lu S., Xu X.Y., Hu S.L., Li B., et al. (2016) Prognostic significance of nuclear or cytoplasmic nucleolin expression in human non-small cell lung cancer and its relationship with DNA-PKcs, Tumour Biol 37 10349-10356.
47. Marcel V., Catez F., Berger C.M., Perrial E., Plesa A. et al. (2017) Expression Profiling of Ribosome Biogenesis Factors Reveals Nucleolin as a Novel Potential Marker to Predict Outcome in AML Patients, PLoS One 12170160.

48. Nguyen Van Long F., Lardy-Cleaud A., Bray S., Chabaud S., Dubois T. et al. (2018) Druggable Nucleolin Identifies Breast Tumours Associated with Poor Prognosis That Exhibit Different Biological Processes, Cancers (Basel) 10.

49. Qiu W., Zhou F., Zhang Q., Sun X., Shi X. et al. (2013) Over expression of nucleolin and different expression sites both related to the prognosis of gastric cancer, APMIS 121 919-925.

50. Peng L., Liang J., Wang H., Song X., Rashid A. et al. (2010) High levels of nucleolar expression of nucleolin are associated with better prognosis in patients with stage II pancreatic ductal adenocarcinoma, Clin Cancer Res 16 3734-3742.

51. Zhao H., Huang Y., Xue C., Chen Y., Hou X. et al. (2013) Prognostic significance of the combined score of endothelial expression of nucleolin and CD31 in surgically resected non-small cell lung cancer, PLoS One 8 e54674.

52. Pichiorri F., Palmieri D., De Luca L., Consiglio J., You J. et al. (2013) In vivo NCL targeting affects breast cancer aggressiveness through miRNA regulation, $J$ Exp Med 210 951-968.

\section{Citation:}

Philippe Bouvet (2019) Nucleolin: a prognostic marker in cancer?. Cancer Stud Ther J Volume 4(4): 1-4. 